\title{
In-space Shape Measurement of Large Planar Structures
}

\author{
T. Talon* and S. Pellegrino* \\ California Institute of Technology, Pasadena, CA 91125
}

\begin{abstract}
A measurement and integration scheme is proposed to estimate the shape of a large planar structure in space. Lightweight sun sensors distributed on the structure measure the local angles relative to the sun. A reconstruction technique is introduced to estimate the shape of the satellite through a decomposition on a function basis. The estimated shape is determined by the coefficients associated with the basis functions which are calculated from the measurements. A trade-off study to analyze the influence of different reconstruction schemes and the position of the sensors is presented. An optimization scheme minimizes the RMS error between the estimated and true shape. An experiment was conducted to show the feasibility and performance of the proposed system at the lab scale. Finally, a simulation of the accuracy of the presented solution on a $60 \mathrm{~m}$ space solar power module is performed. The expected error is $0.7 \mathrm{~mm}$ RMS using sensors every $30 \mathrm{~cm}$.
\end{abstract}

\section{Nomenclature}

$\begin{array}{ll}A & \text { Measurement matrix, rad } \\ K_{\hat{\mathbf{w}}} & \text { Covariance matrix of reconstructed shape, } \mathrm{m}^{2} \\ M & \text { Number of basis functions } \\ \mathbf{n} & \text { Noise of sensors, rad } \\ N & \text { Number of discrete locations where shape is defined } \\ \mathbf{w} & \text { True shape of structure, } \mathrm{m} \\ \hat{\mathbf{w}} & \text { Estimated/Reconstructed shape of structure, } \mathrm{m} \\ q & \text { Coefficient associated with a basis function } \\ \mathbf{q} & \text { Vector of coefficients } q \\ S & \text { Number of sensors on structure } \\ K_{\theta} & \text { Convariant matrix of measurements, } \mathrm{rad}^{2} \\ M S E & \text { Mean Square Error matrix, } \mathrm{m}^{2} \\ R M S E & \text { Root Mean Square Error, } \mathrm{m} \\ \theta & \text { Measurements, rad } \\ \phi & \text { Basis function, } \mathrm{m} \\ \Phi & \text { Matrix of basis functions, } \mathrm{m}\end{array}$

\section{Introduction}

Large planar spacecraft structures are required for applications such as solar sails, solar panels, and large antennas. Traditionally, stiffness driven designs have been adopted for large spacecraft structures [1], but the quest for more aggressive structural concepts, with the potential to achieve much lower areal densities and lower cost through simpler structures, motivates studies of novel concepts for ultralight and highly flexible structures. The Space Solar Power Initiative (SSPI) at Caltech aims to create a phased antenna array on

\footnotetext{
${ }^{*}$ Graduate Student, Graduate Aerospace Laboratories, 1200 East California Boulevard, Mail Code 205-45. e-mail: ttalon@caltech.edu

${ }^{\dagger}$ Professor of Aeronautics and Civil Engineering, Graduate Aerospace Laboratories, 1200 East California Boulevard, Mail Code 105-50, Fellow AIAA. e-mail: sergiop@caltech.edu
} 
a membrane-like structure. This array beams to Earth the energy gathered from photovoltaic elements, in the microwave range. This approach requires the shape of the flexible structure to be measured in space to enable correction, through phase shifting, for the deviation of the position of each antenna forming the phased array [2].

The maximum RMSE on the shape measurement of the spacecraft needs to be a fraction of the operating wavelength of the antenna array. For SSPI the radiation frequency is $10 \mathrm{GHz}$, and this requirement translates into a precision on the order of a millimeter. [3]

Many techniques already exist to measure position in space. One can time-integrate acceleration, twice, to obtain a position measurement, which however is affected by noise in the accelerometers leading to an unbounded increase of the uncertainty of the position estimate. [4] Carrier-Phase Differential GPS is used for precise spacecraft relative navigation [5] but lacks precision in geostationary orbits. Ranging systems have been successfully used in space for precise distance measurements [6]. While their accuracy can be orders of magnitude better than the previously stated requirement, they only measure point-to-point distance. Synthetic aperture radar is used to map the earth with great precision [7]. A large viewing angle is however required to avoid shadows. The same drawback also applies for ranging systems. None of these approaches offers the key features of lightness, time stability, and ability to accurately estimate the shape of very large structures.

We present a method to measure the shape of large planar spacecraft structures compatible with a space solar power satellite. The proposed solution is presented, along with a type of sensors that appears to be very suitable for this application. The mathematical framework to convert a discrete measurement set into a continuous estimated shape for the spacecraft is then explained. A system trade-off is detailed to study the parameters involved in the shape reconstruction scheme and the location of the sensors in order to minimize the RMS error of the estimated shape. The viability of the proposed solution has been confirmed by means of an experiment on a lab-scale structure, which has also provided error bounds for the accuracy achievable with first-generation sensors. Finally, simulations were performed to estimate the accuracy of the presented method on a large $60 \mathrm{~m}$ long structure, representing a full scale SSPI module.

\section{Proposed Solution}

It is proposed to use sun sensors spread on the structure, as shown in Figure 1. Each sensor measures two components of the angle between the normal to the structure and the sun. Since the targeted application for the system is a space solar power spacecraft which is always pointing at the sun to harvest energy, the use of this type of sensors seems appropriate.

Sun sensors are simple devices. While many different architectures exist [8-12], it has been decided to use a design involving a quad-photodiode with an aperture hole on top, as shown in Figure 2a. This solution makes the sensors very light $(0.25 \mathrm{~g}$ for the first-generation sensor described in Section $\mathrm{V})$. When the sensor is pointed towards the Sun, the hole creates a spot on the $2 \times 2$ photosensitive array as shown in Figure $2 \mathrm{~b}$. By differentiating the sum of currents of the photodiodes on the left from the ones on the right, the tangent of the angle $\theta$ can be computed. A similar calculation using the bottom and top photodiodes respectively computes the angle $\beta$. Assuming a zero thickness aperture plate, the relations are

$$
\begin{aligned}
& \frac{2 h \tan \theta}{D}=\frac{\left(I_{1}+I_{2}\right)-\left(I_{3}+I_{4}\right)}{I_{1}+I_{2}+I_{3}+I_{4}} \\
& \frac{2 h \tan \beta}{D}=\frac{\left(I_{1}+I_{4}\right)-\left(I_{2}+I_{3}\right)}{I_{1}+I_{2}+I_{3}+I_{4}}
\end{aligned}
$$

where $I_{k}$ is the current of the $k$ th photodiode, $h$ the distance between the aperture plate and the photosensitive plane, $D$ the width of the aperture hole defined in Figure 2a, and $\theta$ and $\beta$ the two components of the angle from the sun defined in Figure 2b.

To measure the shape of the structure, only one spatial integration is required, as discussed in the next section. The degree of risk of this solution is low: if one sensor fails, the overall shape measurement can still be performed using the information from the other sensors, athough the magnitude of the error will increase. 


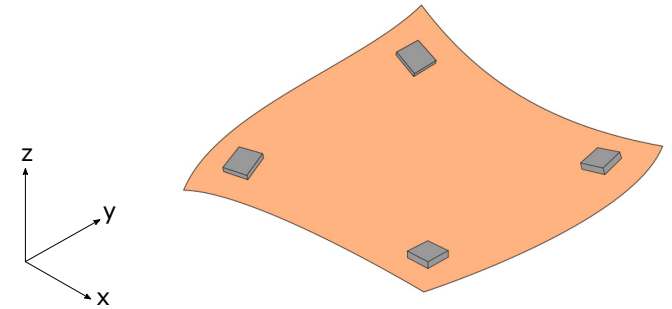

Figure 1: Schematic of proposed solution: 4 sun sensors placed on deformed structure

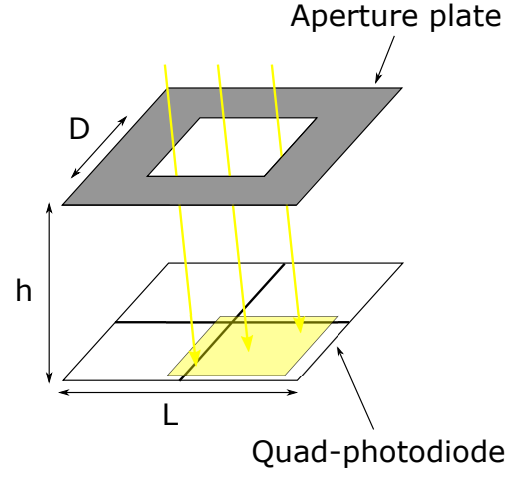

(a) Schematic of sun sensor. $L \approx$ $3-5 \mathrm{~mm}, h \approx 1-2 \mathrm{~mm}$ and $D \approx$ $1-3 \mathrm{~mm}$.

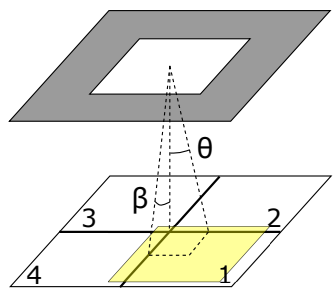

(b) Measured angles and numbering of photodiodes.

Figure 2: Schematic of sun sensor.

\section{Shape Reconstruction}

This section discusses the integration scheme that reconstructs the shape of the structure from the angle measurements. The measured angles correspond to the first derivative of the shape, hence a spatial integration is needed. The shape of the structure, initially flat on the $x y$-plane is defined by the out-of-plane displacement component along the $z$-axis (see Figure 1), w, at $N$ discrete locations. We are looking for an estimated, reconstructed shape of the type

$$
\hat{\mathbf{w}}=\sum_{k=1}^{M} q_{k} \phi_{\mathbf{k}}=\Phi \mathbf{q}
$$

where $\hat{\mathbf{w}} \in \mathbf{R}^{N}$ is the vector of estimated displacements, $q_{k} \in \mathbf{R}$ are unique coefficients, $\phi_{\mathbf{k}} \in \mathbf{R}^{N}$ are the values of basis functions at the discrete locations, $\Phi \in \mathbf{R}^{N \times M}$ is the matrix of basis functions, and $\mathbf{q} \in \mathbf{R}^{M}$ is the vector of basis functions coefficients.

The basis functions need to satisfy the following properties:

1. The whole structure needs to be covered, i.e. for every discrete location $(x, y)$ there is at least one $k$ with $0 \leq k \leq M$ such that $\phi_{\mathbf{k}}(x, y) \neq 0$.

2. They should satisfy at least one Dirichlet boundary condition: the out-of-plane displacement has to be imposed at one location, the starting point for the spatial integration.

3. They must be linearly independent, for the problem to be well-posed and the uniqueness of the coefficients $q_{k}$.

4. They must be differentiable at the sensor locations, for the slopes to be defined at these points.

5. The whole set of sensor measurement needs to be covered, i.e. for every measurement $\theta$ there is at least one $k$ with $0 \leq k \leq M$ such that $\theta \neq 0$ when $\mathbf{w}=\phi_{\mathbf{k}}$. 
6. They must possess sufficient approximation power, i.e. $\hat{\mathbf{w}} \rightarrow \mathbf{w}$ as $M \rightarrow \infty$.

Piecewise polynomial functions or the modal functions (vibration eigenmodes) of the structure are the most obvious choices.

In-order to reconstruct the shape of the structure, one needs to find the coefficients $q_{k}$. They are determined by the angle measurements. The maximum number of coefficients one can calculate is equal to the number of sensor measurements $S$ (equal to twice the number of sensors since each quad-photodiode measures two angles). The reconstructed shape is described by the sum of $M \leq S$ basis functions. The measured angles have to satisfy an equation analogous to Equation 3, and given by:

$$
\theta=A \mathbf{q}+\mathbf{n}
$$

where $\theta \in \mathbf{R}^{S}$ is the vector of the measured angles, $A \in \mathbf{R}^{S \times M}$ are the corresponding angles (or derivatives) of the basis functions at the sensor locations, and called measurement matrix, and $\mathbf{n} \in \mathbf{R}^{S}$ is the noise of the measurements assumed zero mean and Gaussian with covariance $K_{\theta} \in \mathbf{R}^{S \times S}$.

The problem aims to minimize the Mean Squared Error between the reconstructed shape $\hat{\mathbf{w}}$ and the actual true shape $\mathbf{w}$. This error is given by

$$
M S E=K_{\hat{\mathbf{w}}}+(E[\hat{\mathbf{w}}]-\mathbf{w})(E[\hat{\mathbf{w}}]-\mathbf{w})^{T}
$$

where $M S E \in \mathbf{R}^{N \times N}, K_{\hat{\mathbf{w}}} \in \mathbf{R}^{N \times N}$ is the covariance of $\hat{\mathbf{w}}$ and $E[\cdot]$ is the expected value function. $K_{\hat{\mathbf{w}}}$ represents the influence of the sensor noise on the estimated shape. In this least-squares sense, the reconstructed shape follows the same equation as its expected value. From Equations 3 and 4 , w is given by

$$
\hat{\mathbf{w}}=\Phi A^{+} \theta
$$

where $A^{+}$is the Moore-Penrose pseudoinverse of $\mathrm{A}$.

Since the noise of the sensors is assumed Gaussian, a more interesting metric for this problem is the average Root Mean Square (RMS) Error, defined by

$$
R M S E=\sqrt{\frac{\operatorname{Tr}(M S E)}{N}}
$$

where $R M S E \in \mathbf{R}, \operatorname{Tr}$ is the trace function, $\operatorname{Tr}(M S E)$ is the sum of the variance at each discrete location and $M S E$ the Mean Square Error matrix defined by Equation 5.

\section{Minimizing the RMS Error in 1D Case}

For the sake of simplicity, we consider a beam-like structure aligned with the $x$-axis. All results shown is this section can be straightforwardly extended to 2 dimensions.

\section{A. Finite Element approach}

An analysis of the method described in Section III is presented in the context of the finite element method (FEM) [13]. The structure is divided into $M$ elements and nodes. Each node $x_{i}$ has one degree of freedom: its normal displacement component. The basis functions, usually called shape functions, are continuous, piecewise-polynomial, such that

$$
\phi_{k}\left(x_{i}\right)=\delta_{i k} \quad \text { for all } i, k \in\{1, \ldots, M\}
$$

where $\delta_{i k}$ is the Kronecker delta of $i$ and $k$.

We introduce two numbers describing the reconstruction scheme:

1. The order of the element describes the polynomial order of the shape functions and is related to the number of nodes in each element. In $1 \mathrm{D}$, the number of nodes per element equals the order of the element plus 1 .

2. The order of the sensor distribution (or sensor order) describes the number of sensors per element. 
The total number of nodes (discarding the ones used to apply the Dirichlet boundary conditions) and shape functions $M$ is equal to the number of elements times the order of the elements minus the number of boundary conditions plus 1

$$
M=(\# \text { elements }) \times(\text { element order })-(\text { \# Dirichlet boundary conditions })+1
$$

The total number of sensors (or measurements) $S$ is equal to the number of elements times the sensor order

$$
S=(\# \text { elements }) \times(\text { sensor order })
$$

To avoid ill-posed problems, the sensor order needs to be greater or equal to the element order (considering the simple case of a single Dirichlet boundary condition), hence $M \leq S$. Note that adding boundary conditions simply overconstrains the problem.

Figure 3 shows the shape functions for elements and distributions of sensors of order 1 and 2. The sensors and nodes are equally spaced.

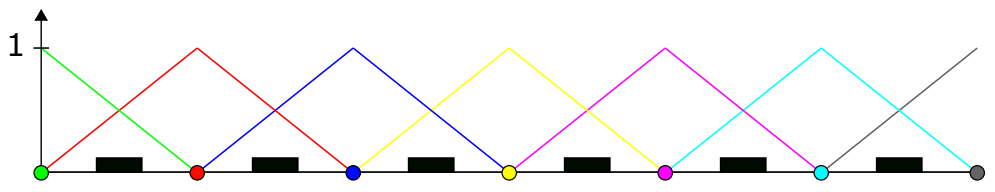

(a) Element order: 1, Sensor order: 1

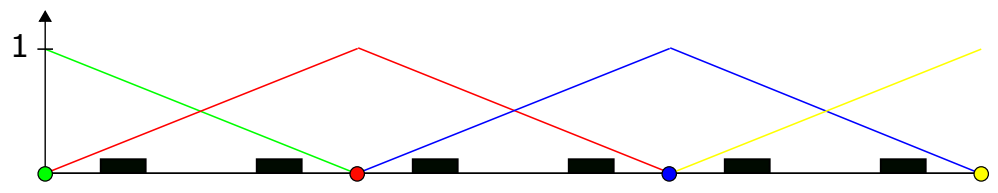

(b) Element order: 1, Sensor order: 2

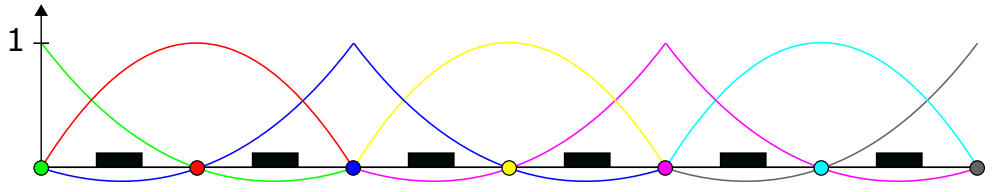

(c) Element order: 2, Sensor order: 2

Figure 3: Finite element shape function for different element and sensor orders. Circles represent the nodes and black rectangles represent the sensors.

For a fixed element order, a larger sensor order will tend to overconstrain the problem, as there are too many sensors for the number of coefficients to calculate. This induces an averaging effect where many measurements are averaged together to calculate a coefficient. This lowers the effect of the noise of the sensors on the calculated coefficient (covariance $K_{\hat{\mathbf{w}}}$ in equation 5 ), but the higher sensor order corresponds to elements of larger size, which decreases the smoothness of the solution and increases the bias (second term in equation 5). Hence, there is a trade-off to be made between the two orders in order to minimize the RMS error.

Figure 4 shows a $30 \mathrm{~m}$ long beam undergoing a sinusoidal deformation of $2 \mathrm{~m}$ amplitude and simply supported at one end. Twenty-four sensors are uniformly distributed and the nodes are equally spaced between the sensors. The $3 \sigma$ RMS error of the sensors is set to $0.017 \mathrm{deg}$ (one arcminute). The results presented in Figure 5 show that for lower element orders the bias is large because the solution is not smooth. For larger element orders the solution is smoother so the bias remains small but the influence of the noise of the sensors increases. The global minimum error is reached when the whole structure is considered as one element, and hence from now on we will consider the structure as one element. Note that this optimization is dependent on the actual shape to be reconstructed and the noise of the sensors. Different shapes will lead to different sets of optimum element/sensor orders while the sensors noise influence the importance of the covariance relative to the bias.

Different global basis functions will be considered from the finite element shape functions introduced in Equation 8, including monomials and modal functions (vibration eigenmodes) of the structure. The latter is an appropriate choice for a large structure in space that vibrates freely. 


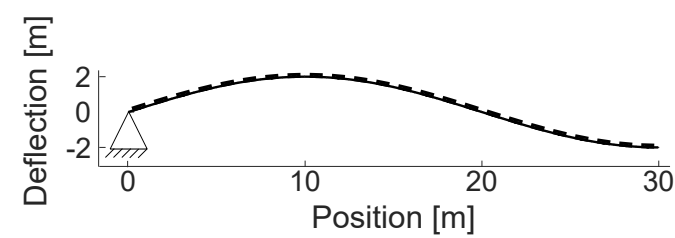

Figure 4: Structure to test finite element reconstruction. Black rectangles represent the sensors.

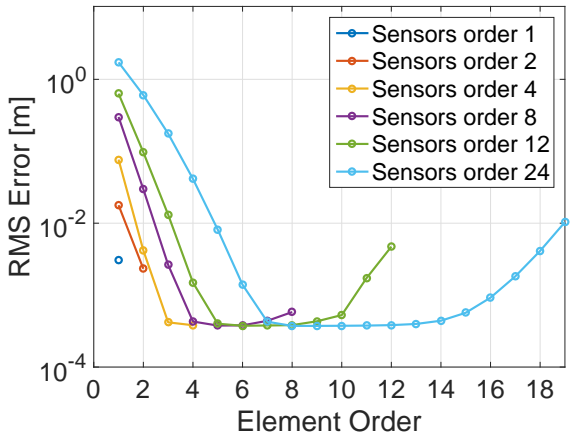

Figure 5: Results of finite element reconstruction of test structure.

\section{B. Sensor Location}

It was shown in Section III that the shape of the structure can be reconstructed from the sensor measurements through the measurement matrix $A$ (Equation 6 ). The noise of each sensor will also propagate to the final solution through a similar equation. The covariance matrix of the position vector $K_{\hat{\mathbf{w}}} \in R^{N \times N}$ is described by

$$
K_{\hat{\mathbf{w}}}=E\left(\hat{\mathbf{w}} \hat{\mathbf{w}}^{T}\right)-E(\hat{\mathbf{w}}) E\left(\hat{\mathbf{w}}^{T}\right)=\Phi A^{+} K_{\theta}\left(A^{+}\right)^{T} \Phi^{T}
$$

Note that if the measurements are independent and have the same noise characteristics, the covariance matrix of the measurement is $K_{\theta}=\sigma_{\theta}^{2} I$, where $\sigma_{\theta}^{2}$ the variance of the measurements and $I \in R^{S \times S}$ the identity matrix. These assumptions are considered valid for this problem.

A good measure to minimize the covariance $K_{\hat{\mathbf{w}}}$ is its trace [14]. This is equivalent to minimizing the average variance of the position vector. Since this covariance is dependent on the position of the sensors through the measurement matrix $A$, these locations can be optimized to find the global minimum of the trace of $K_{\hat{\mathbf{w}}}$.

To solve this minimization problem, our structure is discretized into possible locations for the sensors. A Simulated Annealing algorithm (SA) [15] has been implemented to solve this multivariable, non-convex, global minimization problem. The state is represented by a vector with size equal to the number of candidate positions for the sensors, and filled with ones where it is chosen to locate a sensor, and zeros otherwise. The transition between two steps is done by selecting two random indexes of the state vector and swapping the values in between. From this state, the matrix $A$ is computed to calculate the trace of $K_{\hat{\mathbf{w}}}$ which is used as the cost function. Note that when the sensor order is small, this problem becomes simple and a scanning algorithm that tries every possible combination can be used.

This algorithm was applied to a single element using the finite element shape functions from Equation 8. The distance between each node is constant and equal to $25 \mathrm{~mm}$. Trial positions for the sensors were set every millimeter. The element and sensor order were equal and varied from 2 to 4 . For the first order, the placement of the sensor does not affect the covariance $K_{\hat{\mathrm{w}}}$. The results were compared to the result from a scanning algorithm and are shown in Table 1 . The variance of the sensor measurement $\sigma_{\theta}^{2}$ is set to 1 . Its value does not affect the results of this minimization problem.

The scanning algorithm finds the optimum combination that minimizes the trace of $K_{\hat{\mathbf{w}}}$ (cost) shown in column 2 of Table 1. The third column shows that SA converges to a value close to the global minimum. The location of the sensors is within $2 \mathrm{~mm}$ from the scanning algorithm results which is considered acceptable. The number of calculations is orders of magnitude smaller and increases slowly with the number of sensors. This result becomes useful when hundreds or thousands of sensors need to be positioned on a structure. 
For the three analyzed cases, Figure 6 shows the position of the sensors on the element. For the first case, Figure 6a, the sensors are placed on the edge nodes where the full derivative is not defined (see Figure 3). The considered derivatives are respectively the right and left derivatives for the left and right sensors.

\begin{tabular}{|l|l|l|l|l|}
\hline Order & Scan cost & SA cost & Scan calculations & SA calculations \\
\hline 2 & 6316 & 6350 & 1325 & 95 \\
\hline 3 & 20862 & 20877 & 73149 & 155 \\
\hline 4 & 40246 & 40728 & 4249574 & 75 \\
\hline
\end{tabular}

Table 1: Comparison of simulation results using Scan and SA algorithms for simply supported beam.

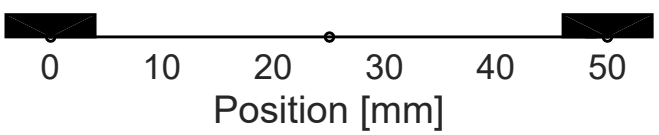

(a) Element order: 2, Sensor order: 2

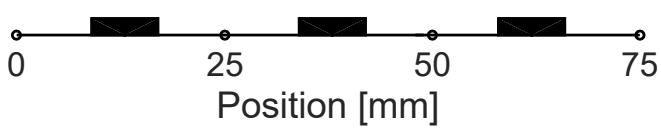

(b) Element order: 3, Sensor order: 3

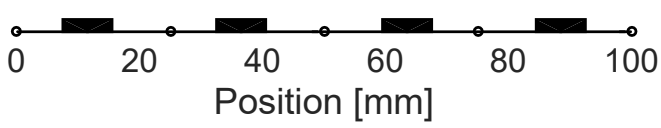

(c) Element order: 4, Sensor order: 4

Figure 6: Optimum location of sun sensors to minimize the influence of their noise on the reconstructed shape using finite element shape functions. Circles correspond to the nodes and rectangles to the sensors.

\section{Experiment}

In order to show the feasibility and performance of the proposed solution, an experiment was conducted.

Sun sensors were made from off-the-shelf quad-photodiodes (TT Electronics OPR5911) with an aperture plate made of a black polymer. This plate was centered on the array of photodiodes under a microscope. No optimization has been made towards the size of the aperture hole $(1.5 \mathrm{~mm}$ diameter $)$ and its height relative to the photosensitive plane $(420 \mu \mathrm{m})$. These two parameters dictate the field of view and sensitivity of the sensor. Note that it is possible to achieve an maximum sensitivity for a fixed field of view. This lowers the influence of the current noise from each photodiode to the angle calculation and hence, lowers the sensor angle noise.

The structure is a $30 \mathrm{~cm}$ by $5 \mathrm{~cm}, 50 \mu \mathrm{m}$ thick Kapton strip. Silver traces were printed on the back side to connect the photodiodes to an electronic circuit. This circuit was not located on the structure itself. The sensors were placed on the front side and connected to the traces through small holes filled with conductive epoxy. The epoxy provides electrical connection as well as mechanical bonding between the sensors and the structure. The position of each sensor was optimized to reconstruct the shape using a 3rd order polynomial function and with basis functions $x, x^{2}, x^{3}$.

The Kapton strip was clamped at the top, where the wires were bonded with conductive epoxy to the silver traces. The wires connect to an electrical circuit to convert the current from the photodiodes to a voltage readable by an Arduino board (Arduino Due). The structure hangs under gravity and is deformed by a sliding plate placed at about $23 \mathrm{~cm}$ from the top.

A white diode was placed $30 \mathrm{~cm}$ away from the structure, to illuminate the sensors. A reference measurement of the structure was performed with the Vic3D Digital Image Correlation (DIC) system. The DIC measurements were used for comparison with the reconstructed shape and hence to estimate the performance of our measurement and reconstruction technique. A pattern of white dots on a black background was spray painted on the front side of the structure to use this system. 


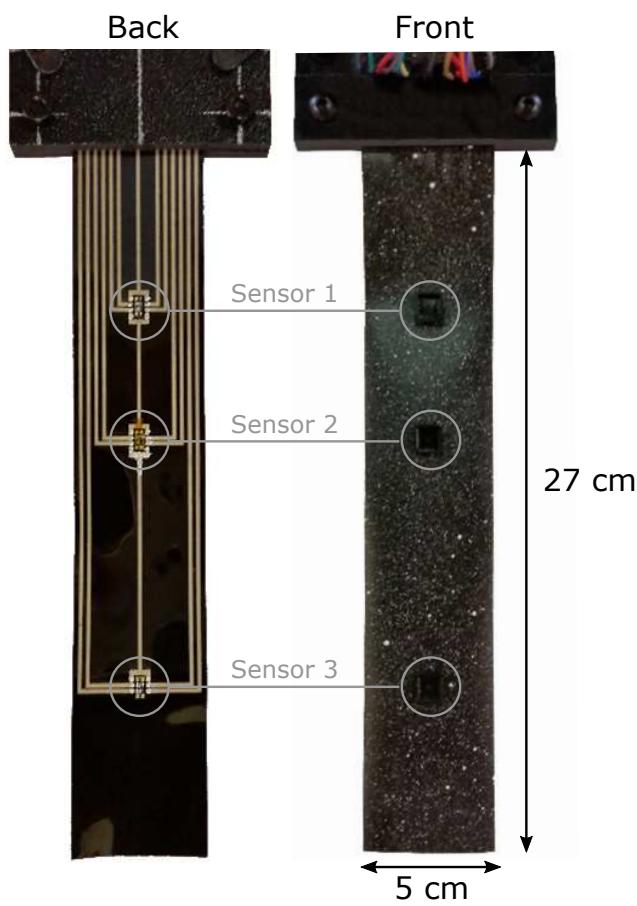

Figure 7: Structure for the experiment: Kapton strip with silver traces on the back, painted black with white spots on the front.

The first step of the experiment was to calibrate each sensor, i.e. to find the relationship between the angle of each sensor and its output. This was performed by applying 5 different shapes to the structure. While the measurements are directly provided by the sensors, the angles are measured by the DIC. The DIC system also provides a measurement of the position of each sensor relative to the light to account for the bias created by the finite distance of the source. The five data points were fitted by a piecewise linear function. The calibration also provided information about the noise of each sensor (in a standard deviation sense). A conservative number for the error is $3 \sigma=0.15 \mathrm{deg} @ 1 \mathrm{~Hz}$.

A different shape was imposed for the actual test. To account for the finite distance of the light source, a loop was included in the reconstruction algorithm: a first shape is reconstructed and from the estimated deflections, the position of each sensor is calculated. Then, the angles are corrected and fed back to calculate a new shape. This loop is repeated until the difference between two consecutive reconstructed shapes is smaller than a specified threshold.

The results are shown Figure 8. A third-order polynomial was used to recreate the shape of the structure (element and sensor of order 3). The final RMS error between the estimated shape and the DIC measurement was $0.2 \mathrm{~mm}$.

This result confirms that our preliminary test apparatus and shape reconstruction tool are appropriate to study this problem.

\section{Simulation of Large-Scale Structure}

Simulations were carried out to estimate the performance of the proposed solution on a large structure, which simulates a $60 \mathrm{~m}$ long and $1.5 \mathrm{~m}$ wide strip for an SSPI spacecraft.

The model is a 3D Euler-Bernoulli beam simply supported at both ends. 1000 sensors were uniformly distributed on the structure, on a square grid with size of $30 \mathrm{~cm}$. Their effects on the mass and stiffness of the beam were neglected. The noise in the angle measurements was considered to be $0.15 \mathrm{deg}$ RMS @ 1 $\mathrm{Hz}$ as derived from the experiment in Section V. Sensors measure two angles, as described in Figure $2 \mathrm{~b}$, to capture both out-of-plane bending and torsion of the strip.

The strip was deformed to the shape of its first vibration mode with an amplitude of $50 \mathrm{~cm}$. The variation of each sensor angle was numerically simulated every 1 sec. Since the frequency of the first mode $(0.004$ 


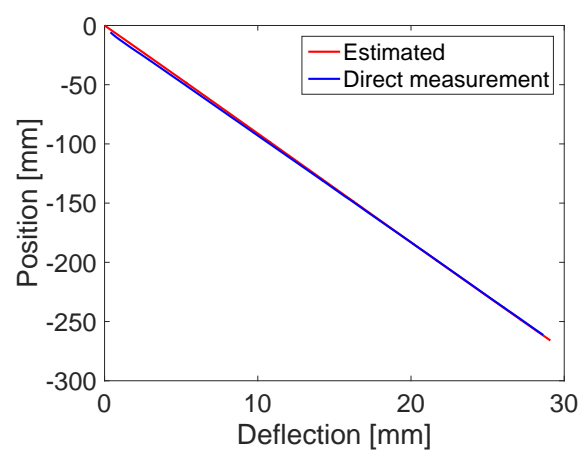

(a) Deflection calculated from the DIC (blue) and the reconstruction from the sensors (red).

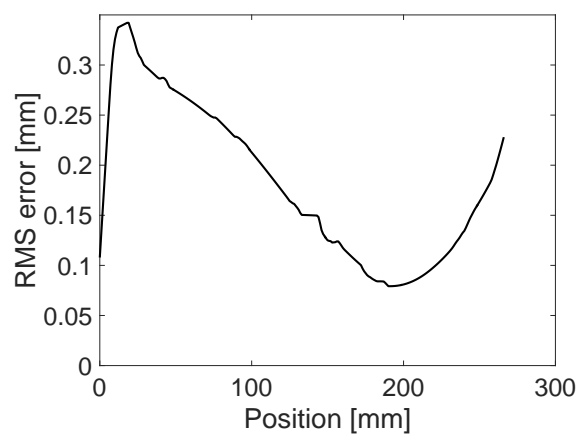

(b) Evolution of the RMS error along the strip. The large initial error is due to the effect of the clamp flattening the structure at the top.

Figure 8: Results of the experiment

$\mathrm{Hz}$ ) is orders of magnitude lower than the bandwidth of the measurement, the shape is considered static. The matrices $\Phi$ (Equation 3) and $A$ (Equation 4) were precomputed from a finite element model of the structure. The integration scheme considered a single element (sensor order of 1000) of order 100. The basis functions were the first 50 bending modes and the first 50 torsional modes calculated from the finite element model. the discrete locations where the shape is computed form a grid of size $10 \mathrm{~cm}$. Using Equation 6, the reconstructed shape was calculated and then compared to the truth, i.e. the analytical shape of the first mode.

Figure 9 shows the root mean square error as a function of the position on the strip. The error is defined at each time step as the difference between the estimated shape and the truth for each discrete location as in Equations 5 and 7. The RMS error reaches a maximum of $0.8 \mathrm{~mm}$ at the center of the strip and is equal to $0.7 \mathrm{~mm}$ on average. If a phased antenna array working at $10 \mathrm{GHz}$ were to be placed on this structure, this would correspond to a loss of efficiency of $2.2 \%$. [3]

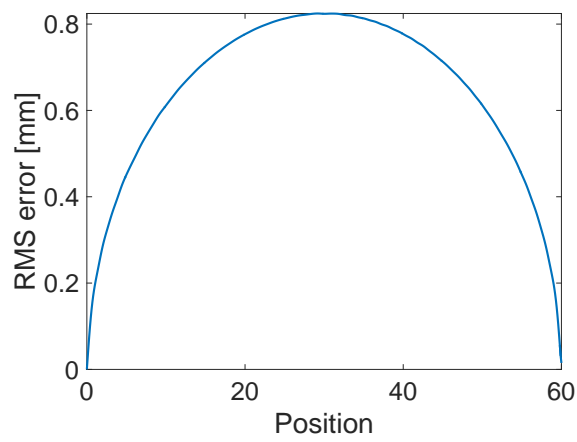

Figure 9: Root mean square error between the estimated and the true shape along a $60 \mathrm{~m}$ strip

\section{Conclusion}

A technique to use distributed sensors to measure the shape of a large flexible structure in space has been proposed. Different integration schemes have been analyzed and the existence of optimum parameters to minimize the RMS error of the reconstructed shape has been established. Our final approach combines measurements from sun sensors spread on a large area satellite with a model of its dynamics, to identify the contribution of each vibration mode on the current shape and estimate its magnitude. An experiment was conducted to validate the performance of such a system and our prediction tools. The RMS error obtained on a 1D, $30 \mathrm{~cm}$ long structure with 3 sensors was $0.2 \mathrm{~mm}$; matching our predictions. Simulations were programmed to estimate the performance of this system on a large-scale structure. A RMS error of $0.7 \mathrm{~mm}$ 
was predicted which is within the requirements of the Space Solar Power Initiative.

\section{Acknowledgment}

This research was supported by the Northrop Grumman Corporation.

\section{References}

${ }^{1}$ Hedgepeth, J. M., Critical requirements for the design of large space structures, Vol. 3484, National Aeronautics and Space Administration, Scientific and Technical Information Branch, 1981.

${ }^{2}$ Wang, H. and Hajimiri, A., "A wideband CMOS linear digital phase rotator," 2007 IEEE Custom Integrated Circuits Conference, IEEE, 2007, pp. 671-674.

${ }^{3}$ Bakr, O. and Johnson, M., "Impact of phase and amplitude errors on array performance," EECS Department, University of California, Berkeley, Tech. Rep. UCB/EECS-2009-1, 2009.

${ }^{4} \mathrm{Zhu}$, W. H. and Lamarche, T., "Velocity estimation by using position and acceleration sensors," Industrial Electronics, IEEE Transactions on, Vol. 54, No. 5, 2007, pp. 2706-2715.

${ }^{5}$ Psiaki, M. L. and Mohiuddin, S., "Modeling, analysis, and simulation of GPS carrier phase for spacecraft relative navigation," Journal of Guidance, Control, and Dynamics, Vol. 30, No. 6, 2007, pp. 1628-1639.

${ }^{6}$ Tapley, B. D., Bettadpur, S., Watkins, M., and Reigber, C., "The gravity recovery and climate experiment: Mission overview and early results," Geophysical Research Letters, Vol. 31, No. 9, 2004, L09607.

${ }^{7}$ Curlander, J. C. and McDonough, R. N., Synthetic aperture radar, J. Wiley \& Sons New York, NY, USA, 1991.

${ }^{8}$ Chen, F. and Feng, J., "Analogue sun sensor based on the optical nonlinear compensation measuring principle," Measurement Science and Technology, Vol. 18, No. 7, 2007, pp. 2111.

${ }^{9}$ Zaffanella, C., Savoca, R., and Bednarek, T., "Analog sun sensor," Nov. 5 1996, US Patent 5,572,316.

${ }^{10}$ Shapiro, W., "Digital sun sensor having sub-image resolution," April 17 1973, US Patent 3,728,715.

${ }^{11}$ Okamoto, T., "Digital sun sensor," Oct. 17 1989, US Patent 4,874,937.

${ }^{12}$ Delgado, F. J., Quero, J. M., Garcia, J., Tarrida, C. L., Ortega, P. R., and Bermejo, S., "Accurate and wide-field-of-view MEMS-based sun sensor for industrial applications," Industrial Electronics, IEEE Transactions on, Vol. 59, No. 12, 2012, pp. $4871-4880$

${ }^{13}$ Zienkiewicz, O. C. and Taylor, R. L., The finite element method, Vol. 3, McGraw-hill London, 1977.

${ }^{14}$ Beck, J. and Arnold, K., Parameter Estimation in Engineering and Science, Probability and Statistics Series, Wiley, 1977.

${ }^{15}$ Hwang, C. R., "Simulated annealing: theory and applications," Acta Applicandae Mathematicae, Vol. 12, No. 1, 1988, pp. $108-111$. 